\title{
RESOLUTIONS AND PERIODICITY
}

\author{
J. ALPERIN ${ }^{1}$ AND G. JANUSZ ${ }^{2}$
}

ABSTRACT. The representation theory of groups with a cyclic Sylow subgroup is used to give a detailed construction of minimal projective resolutions.

The Artin-Tate theorem [1, p. 262] has as a consequence that a Sylow $p$-subgroup of a finite group $G$, for an odd prime $p$, is cyclic exactly when the cohomology $H^{n}\left(G, Z_{p}\right)$ is periodic for positive integers $n$. Moreover, in this case Swan [4] has shown that the minimal projective resolution of $Z_{p}$ over $Z_{p}[G]$ is periodic. By using the deep theory of groups with a cyclic Sylow subgroup we can go one step further.

THEOREM. If $G$ is a finite group with a cyclic Sylow p-subgroup for a prime $p$ and $F$ is a splitting field of characteristic $p$ for $G$ then each term of the minimal projective resolution of $F$ over $F[G]$ is indecomposable.

Actually, we shall prove much more, but the indecomposability seems to be the most important conclusion. To describe our results fully we need some notation. Let $e=\left|N\left(S_{p}\right): C\left(S_{p}\right)\right|$, where $S_{p}$ is a Sylow $p$-subgroup of $G$, and $V_{1}=F, V_{2}, \cdots, V_{e}$ be irreducible $F[G]$ modules in the principal p-block $B_{0}$ of $G$, one from each isomorphism class of such modules. Let $U_{i}$ be an indecomposable projective $F[G]$ module which has $V_{i}$ as a homomorphic image so that $U_{i}$ is determined to within isomorphism. Finally, let $T$ be the Brauer tree corresponding to $B_{0}$ so that the vertices are in one-to-one correspondence with the $p$-conjugacy classes of ordinary irreducible characters in $B_{0}$ while the edges correspond one-to-one with the isomorphism classes of irreducible $F[G]$ modules in $B_{0}$. Hence, if $E$ is an edge, let $V_{E}=V_{i}$ when the isomorphism class of $V_{i}$ corresponds to $E$. In this case, we also set $U_{E}=U_{i}$, the corresponding projective. Each vertex $P$ of $T$ is assigned a multiplicity $m(P)$; it is known that $m(P)>1$ for at most one vertex $P$. Moreover, there is a canonical circular ordering of the edges emanating from $P$ determined by the structure of the

Received by the editors April 26, 1972.

AMS (MOS) subject classifications (1969). Primary 2050, 2080.

Key words and phrases. Resolution, periodicity, Brauer tree.

1 Supported in part by National Science Foundation grants GP-20864 and GP-28700.

2 Supported in part by National Science Foundation grant GP-21335. 
indecomposable projective $F[G]$ modules in the following way. The projective $U_{E}$ has a unique maximal submodule $M_{E}$ which is in turn a sum

$$
M_{E}=K_{E}(P)+K_{E}(Q),
$$

in which $K_{E}(P)$ and $K_{E}(Q)$ are uniserial modules and $P$ and $Q$ are the vertices on $E=(P Q)$. The module $K_{E}(P)$ has a unique composition series and the composition factors (from the "top down") are $V_{E_{1}}, V_{E_{2}}, \cdots, V_{E}$, where $E_{1}, E_{2}, \cdots, E$ are all the edges on $P$ and the initial sequence of distinct $V$ 's is repeated $m(P)$ times. A similar statement holds for the vertex $Q$. The circular ordering on the edges of $P$ is determined by the above sequence in which $E_{1}$ follows $E$. The intersection $K_{E}(P) \cap K_{E}(Q)=S(E)$ is the unique minimal submodule of $U_{E}$ and is isomorphic with $V_{E}$.

We shall now describe a canonical infinite sequence of edges of $T$. Let $E_{0}$ be the unique edge connected to the vertex $P_{0}$ corresponding to the principal character and let $P_{1}$ be the other vertex connected to $E_{0}$. Suppose now that we have defined edges $E_{0}, \cdots, E_{n}$ and vertices $P_{0}, P_{1}, \cdots, P_{n+1}$; let $E_{n+1}$ be the edge immediately following $E_{n}$ in the circular ordering around $P_{n+1}$ and let $P_{n+2}$ be the other vertex connected to $E_{n+1}$. Since $T$ is a tree with exactly $e$ edges it follows readily that in the sequence $E_{0}, \cdots$, $E_{2 e-1}$ each edge appears exactly twice and that $E_{i+2 e}=E_{i}$ for all $i$.

THEOREM 1. If $G$ is a finite group with cyclic Sylow p-subgroup, $F$ is a splitting field of characteristic $p$ for $G$ and

$$
\rightarrow X_{n+1} \rightarrow X_{n} \rightarrow \cdots \rightarrow X_{1} \rightarrow X_{0} \rightarrow F \rightarrow 0
$$

is the minimal projective resolution for $F$ over $F[G]$, then $X_{i} \simeq U_{E_{i}}, i>0$, where $E_{i}$ is the ith edge in the canonical sequence of edges of the Brauer tree for the principal p-block of $G$.

In view of the remarks above, the nature of the period $2 e$ is "explained". A similar result, in which certain $p$-adic rings of characteristic zero replace $F$, is now immediate.

As a first step in the rather short proof we establish the following

Lemma. Let $P$ be a vertex of $T, E$ and $F$ consecutive edges on $P$ and let $Q$ be the other vertex connected to $F$. There exists an exact sequence

$$
0 \rightarrow K_{F}(Q) \rightarrow U_{F} \rightarrow K_{E}(P) \rightarrow 0 .
$$

ProOF. The module $K_{E}(P)$ is uniserial with "top" composition factor isomorphic to $V_{F}$, since $F$ follows $E$ in the ordering around $P$. Thus, $K_{E}(P)$ is a homomorphic image of $U_{F}$. The kernel of this map contains the socle $S(F)$ of $U_{F}$ since $K_{E}(P)$ is not projective and so is not isomorphic to $U_{F}$. Moreover, this kernel must contain $K_{F}(Q)$ because none of the composition factors of $K_{F}(Q) / S(F)$ are composition factors of $K_{E}(P)$. The 
composition lengths of $U_{F} / K_{F}(Q)$ and $K_{E}(P)$ are each equal to the product of $m(P)$ and the number of edges on $P$, so the lemma follows.

Proof (oF Theorem 1). Let $E_{0}=\left(P_{0} P_{1}\right), E_{1}=\left(P_{1} P_{2}\right), \cdots$ be the canonical sequence of edges. Iterated application of the lemma yields the exact sequence

$$
\cdots \rightarrow U_{E_{2}} \rightarrow U_{E_{1}} \rightarrow U_{E_{0}} \rightarrow K_{E_{0}}\left(P_{0}\right) \rightarrow 0
$$

in which the image of $U_{E_{n+1}}$ in $U_{E_{n}}$ is $K_{E_{n}}\left(P_{n}\right)$. But $m\left(P_{0}\right)=1$ and $E_{0}$ is the unique edge connected to $P_{0}$ so $K_{E_{0}}\left(P_{0}\right)$ is the minimal submodule of $U_{E_{0}}$ and $K_{E}\left(P_{0}\right) \simeq F$. Hence, we have obtained a resolution for $F$, which is clearly minimal since each term is indecomposable.

Minimal resolutions of certain other irreducible modules can be similarly described. If the module $V_{E}$ corresponds to the edge $E=(P Q)$ with $E$ the only edge connected to $P$ and $m(P)=1$ then the theorem holds as stated for the minimal resolution of $V_{E}$ using a canonical sequence starting with $E_{0}=E$ and $P_{0}=P$. Again the period is $2 e$. It is known that $E$ satisfies these conditions if and only if $V_{E}$ is the reduction modulo $p$ of an $R[G]$ module for a suitable $p$-adic ring $R$ of characteristic zero (see Corollary 7.3 of [2]). When $E$ is an edge not satisfying these conditions, the minimal projective resolution of $V_{E}$ will always contain decomposable terms and appears to be considerably more difficult to describe.

For example, let $G=\operatorname{PSL}(2, p)$ with $p$ a prime exceeding three. In this case $e=\frac{1}{2}(p-1)$ and the Brauer tree is an open polygon with edges

$$
E_{0}=\left(P_{0} P_{1}\right), E_{1}=\left(P_{1} P_{2}\right), \cdots, E_{e-1}=\left(P_{e-1} P_{e}\right)
$$

and where $m\left(P_{e}\right)=2$. The minimal projective resolution for $V_{E_{e-1}}$ contains a term which is the sum of $e$ indecomposable projectives. However, this is as "bad" as the situation can get, as we shall now show.

TheOREM 2. Let $G$ and $F$ be as in Theorem 1 and let $M$ be any indecomposable $F[G]$ module. If the minimal projective resolution for $M$ over $F[G]$ is

$$
\rightarrow X_{n} \rightarrow \cdots \rightarrow X_{1} \rightarrow X_{0} \rightarrow M \rightarrow 0
$$

then $X_{i}, i \geqq 0$, has no repeated indecomposable summand. In particular, $X_{i}$ is the sum of at most e indecomposable $F[G]$ modules where $e$ is the number of irreducible $F[G]$ modules in the p-block containing $M$.

Proof. Let $X_{-1}=M$ and let $D_{i}, i \geqq 0$, be the kernel of the map from $X_{i}$ to $X_{i-1}$. Since $M$ is indecomposable and the resolution is minimal, it follows that $D_{0}$ is indecomposable [3]. Iterating this argument we deduce that each $D_{i}$ is indecomposable, $i \geqq 0$. By Corollary 7.7 of [2] the socle of $D_{i}$ is multiplicity free. The minimality of the resolution guarantees that the socle of $D_{i}$ and the socle of $X_{i}$ coincide. The theorem follows. 


\section{REFERENCES}

1. H. Cartan and S. Eilenberg, Homological algebra, Princeton Univ. Press, Princeton, N.J., 1956, MR 17, 1040; MR 22 \#045.

2. G. J. Janusz, Indecomposable modules for finite groups, Ann. of Math. (2) 89 (1969), 209-241. MR 39 \#5622.

3. A. Heller, Indecomposable representations and the loop-space operation, Proc. Amer. Math. Soc. 12 (1961), 640-643. MR 23 \#A3778.

4. R. G. Swan, Periodic resolutions for finite groups, Ann. of Math. (2) 72 (1960), 267-291. MR 23 \#A2205.

Department of Mathematics, University of Chicago, Chicago, Illinois 60637

Department of Mathematics, University of Illinois, Urbana, Illinois 61801 